\title{
GLOBAL SOLUTIONS OF THE 2D DISSIPATIVE QUASI-GEOSTROPHIC EQUATION IN BESOV SPACES*
}

\author{
JIAHONG WU
}

\begin{abstract}
The two-dimensional (2D) quasi-geostrophic (QG) equation is a 2D model of the 3D incompressible Euler equations, and its dissipative version includes an extra term bearing the operator $(-\Delta)^{\alpha}$ with $\alpha \in[0,1]$. Existing research appears to indicate the criticality of $\alpha=\frac{1}{2}$ in the sense that the issue of global existence for the 2D dissipative QG equation becomes extremely difficult when $\alpha \leq \frac{1}{2}$. It is shown here that for any $\alpha \leq \frac{1}{2}$ the $2 \mathrm{D}$ dissipative QG equation with an initial datum in the Besov space $B_{2, \infty}^{r}$ or $B_{p, \infty}^{r}(p>2)$ possesses a unique global solution if the norm of the datum in these spaces is comparable to $\kappa$, the diffusion coefficient. Since the Sobolev space $H^{r}$ is embedded in $B_{2, \infty}^{r}$, a special consequence is the global existence of small data solutions in $H^{r}$ for any $r>2-2 \alpha$.
\end{abstract}

Key words. 2D quasi-geostrophic equation, Besov spaces, global existence

AMS subject classifications. 76U05, 76B03, 35Q35

DOI. $10.1137 /$ S0036141003435576

1. Introduction. This paper is concerned with global existence results for the two-dimensional (2D) dissipative quasi-geostrophic (QG) equation

$$
\left\{\begin{array}{l}
\partial_{t} \theta+u \cdot \nabla \theta+\kappa(-\Delta)^{\alpha} \theta=0, \\
u=\left(u_{1}, u_{2}\right)=\nabla^{\perp} \psi, \quad(-\Delta)^{\frac{1}{2}} \psi=\theta
\end{array}\right.
$$

supplemented with the initial condition

$$
\theta(x, 0)=\theta_{0}(x)
$$

In (1.1), $x \in \mathbb{R}^{2}, t \geq 0, \kappa>0$ is the diffusion coefficient and $\alpha \in[0,1]$ is a parameter, $\theta=\theta(x, t)$ is a scalar representing the temperature, $u$ is the velocity field, and $\psi$ is the usual stream function. Besides its geophysical applications [11], [12], the 2D dissipative QG equation serves as a 2D model of the 3D Navier-Stokes equations and has recently been extensively investigated (see [1], [2], [3], [5], [6], [7], [8], [9], [10], [13], [14], [15], [16]).

Prior work on the issue of global existence concerning the 2D dissipative QG equation (1.1) appears to indicate that $\alpha=\frac{1}{2}$ is a critical index. In the subcritical case, namely, $\alpha>\frac{1}{2}$, solutions at several regularity levels, including solutions in the classical sense, have been shown to be global in time [7], [13], [16]. The theory of global existence and regularity for this case is thus in a satisfactory state. In the critical case $\alpha=\frac{1}{2}$, classical solutions are known to be global if their initial $L^{\infty}$-norms are comparable to $\kappa[6]$. For initial data of arbitrary size, the global existence of classical solutions has not been established. It is hoped that the resolution of this problem will shed light on the millennium prize problem on the 3D Navier-Stokes equations. The supercritical case $\alpha<\frac{1}{2}$ seems even harder to deal with, and work on this case has just started to appear. For $\alpha \leq \frac{1}{2}$, Chae and Lee [3] established a global existence

\footnotetext{
* Received by the editors September 29, 2003; accepted for publication (in revised form) May 28, 2004; published electronically January 5, 2005.

http://www.siam.org/journals/sima/36-3/43557.html

${ }^{\dagger}$ Department of Mathematics, Oklahoma State University, Stillwater, OK 74078 (jiahong @math.okstate.edu).
} 
result under the assumption that $\theta_{0}$ is small in the Besov space $B_{2,1}^{2-2 \alpha}$. In a recent work [9], A Córdoba and D. Córdoba obtained for any $\alpha \in[0,1]$ a local existence result for $\theta_{0} \in H^{s}$ with $s+\alpha>2$ and a global result for small $\theta_{0}$ in $H^{s}$ with $s>2$ or in $H^{3 / 2}$ in the case of $\alpha=\frac{1}{2}$.

This paper is devoted to establishing global existence results for (1.1) with $\theta_{0}$ in the Besov space $B_{2, \infty}^{r}$ or in $B_{p, \infty}^{r}$ with $p>2$. For any $\alpha \leq \frac{1}{2}$ and $\theta_{0} \in B_{2, \infty}^{r}$ with $r>2-2 \alpha$, we prove that the 2D QG equation (1.1) has a unique global solution provided that the norm of $\theta_{0}$ in $B_{2, \infty}^{r}$ is comparable to $\kappa$. Because of the embeddings $B_{2,1}^{r} \hookrightarrow H^{r} \hookrightarrow B_{2, \infty}^{r}$, a special consequence is the global existence result for small data in $B_{2,1}^{r}$ or $H^{r}$ with $r>2-2 \alpha$. We defer the precise statement and many more details to section 3 .

The situation for $\theta_{0} \in B_{p, \infty}^{r}$ with $p>2$ is more sophisticated and the major difficulty lies in how to obtain suitable lower bounds for terms generated from the dissipative part. Thanks to the $L^{p}$-decay estimate of A. Córdoba and D. Córdoba [9], we are able to establish a global existence result for solutions in the Besov space $B_{p, \infty}^{r}$ with $r>1+\frac{2}{p}$. Appropriate smallness conditions are imposed on the initial datum $\theta_{0}$ here. This is accomplished in section 4, which consists of two subsections. The first subsection provides an a priori bound and the second proves the global existence result.

2. Preliminaries. This section provides a precise characterization of the Besov space $B_{p, q}^{r}$ through the Littlewood-Paley decomposition and gathers several important estimates involving $B_{p, \infty}^{r}$. First, we recall two commutator estimates established in a previous work [17]. Then follows the tame estimate for the usual product of two functions. Finally, a logarithmic bound for the $L^{\infty}$-norm of a function in terms of its norms in Besov spaces is stated and proven. We shall also reproduce here the $L^{p}$-decay estimate of A. Córdoba and D. Córdoba for the dissipative QG equation [9].

We start with a dyadic decomposition of $\mathbb{R}^{d}$, where $d>0$ is an integer. It is a classical result that there exist two radial functions $\chi \in C_{0}^{\infty}\left(\mathbb{R}^{d}\right)$ and $\phi \in C_{0}^{\infty}\left(\mathbb{R}^{d} \backslash\{0\}\right)$ satisfying

$$
\begin{gathered}
\operatorname{supp} \chi \subset\{\xi:|\xi| \leq 4 / 3\}, \quad \operatorname{supp} \phi \subset\{\xi: 3 / 4<|\xi|<8 / 3\}, \\
\chi(\xi)+\sum_{j \geq 0} \phi\left(2^{-j} \xi\right)=1 \quad \text { for all } \xi \in \mathbb{R}^{d} .
\end{gathered}
$$

For the purpose of isolating different Fourier frequencies, define the operators $\Delta_{i}$ for $i \in \mathbb{Z}$ as follows:

$$
\Delta_{i} u= \begin{cases}0 & \text { if } i \leq-2, \\ \chi(D) u=\int h(y) u(x-y) d y & \text { if } i=-1, \\ \phi\left(2^{-i} D\right) u=2^{i d} \int g\left(2^{i} y\right) u(x-y) d y & \text { if } i \geq 0\end{cases}
$$

where $h=\chi^{\vee}$ and $g=\phi^{\vee}$ are the inverse Fourier transforms of $\chi$ and $\phi$, respectively. We note that $\Delta_{i}$ in (2.1) can be defined in other ways. For example, by further requiring $\chi(\xi)=1$ for $|\xi| \leq \frac{3}{8}$ and writing

$$
g(x)=2^{d} h(2 x)-h(x), \quad g_{j}(x)=2^{d j} g\left(2^{j} x\right),
$$

one can define $\Delta_{-1}=h *$ and $\Delta_{j}=g_{j} *$ for $j \geq 0$. 
For $i \in \mathbb{Z}, S_{i}$ is the sum of $\Delta_{j}$ with $j \leq i-1$, i.e.,

$$
S_{i} u=\Delta_{-1} u+\Delta_{0} u+\Delta_{1} u+\cdots+\Delta_{i-1} u=\int_{\mathbb{R}^{d}} h\left(2^{i} y\right) u(x-y) d y .
$$

It can be shown for any tempered distribution $f$ that $S_{i} f \rightarrow f$ in the distributional sense, as $i \rightarrow \infty$.

For any $r \in \mathbb{R}$ and $p, q \in[1, \infty]$, the Besov space $B_{p, q}^{r}$ consists of all tempered distributions $f$ such that the sequence $\left\{2^{j r}\left\|\Delta_{j} f\right\|_{L^{p}}\right\}_{j \in \mathbb{Z}}$ belongs to $L^{q}(\mathbb{Z})$. In particular, $B_{p, \infty}^{r}$ contains any function $f$ satisfying

$$
\|f\|_{B_{p, \infty}^{r}} \equiv \sup _{j \in \mathbb{Z}} 2^{j r}\left\|\Delta_{j} f\right\|_{L^{p}}<\infty .
$$

It is easy to check that $B_{p, \infty}^{r}$ endowed with the norm (2.2) is a Banach space.

The following version of Bernstein's lemma can be found in [4].

Lemma 2.1 (Bernstein's lemma). Let $d>0$ be an integer and $R_{2}>R_{1}>0$ be two real numbers. If $p \in[1, \infty]$ and $\operatorname{supp} \widehat{f} \subset\left\{\xi \in \mathbb{R}^{d}: R_{1} 2^{j} \leq|\xi| \leq R_{2} 2^{j}\right\}$, then

$$
C^{-1} 2^{j k}\|f\|_{L^{p}\left(\mathbb{R}^{d}\right)} \leq \max _{|\alpha|=k}\left\|\partial^{\alpha} f\right\|_{L^{p}\left(\mathbb{R}^{d}\right)} \leq C 2^{j k}\|f\|_{L^{p}\left(\mathbb{R}^{d}\right)},
$$

where $C>0$ is a constant depending on $k, R_{1}$, and $R_{2}$ only.

We now recall two commutator estimates previously established in [17].

Proposition 2.2. Let $j \geq-1$ be an integer, let $r \in \mathbb{R}$, and let $p \in[1, \infty]$. Then,

$$
\left\|\left[u \cdot \nabla, \Delta_{j}\right] \theta\right\|_{L^{p}} \leq C 2^{-j r}\left(\|\nabla \theta\|_{L^{\infty}}\|u\|_{B_{p, \infty}^{r}}+\|\nabla u\|_{L^{\infty}}\|\theta\|_{B_{p, \infty}^{r}}\right),
$$

where $C$ is a pure constant and the brackets [, ] represent the commutator, namely,

$$
\left[u \cdot \nabla, \Delta_{j}\right] \theta=u \cdot \nabla\left(\Delta_{j} \theta\right)-\Delta_{j}(u \cdot \nabla \theta) .
$$

Inequality (2.3) is suitable for situations when $u$ and $\theta$ are equally regular. If $\nabla \theta$ is not known to be bounded in $L^{\infty}$, then (2.3) fails. The following proposition provides a new estimate which needs no information about $\nabla \theta$. As a trade-off, $u$ is required to be in $B_{p, \infty}^{r+1}$. The importance of this estimate will be seen in the proofs of Theorems 3.1 and 4.1 .

Proposition 2.3. Let $j \geq-1$, let $r \in \mathbb{R}$, and let $p \in[1, \infty]$. Then, for some pure constant $C$,

$$
\left\|\left[u \cdot \nabla, \Delta_{j}\right] \theta\right\|_{L^{p}} \leq C 2^{-j r}\left(\|\nabla u\|_{L^{\infty}}\|\theta\|_{B_{p, \infty}^{r}}+\|\theta\|_{L^{\infty}}\|u\|_{B_{p, \infty}^{r+\infty}}\right) .
$$

Estimates for the product $u v$ of two functions $u$ and $v$ are handy in dealing with the quadratic nonlinear term in many partial differential equations. In the context of Besov spaces, we have the following estimate.

Proposition 2.4. Let $r>0$ be a real number and let $p \in[1, \infty]$. Then

$$
\|u v\|_{B_{p, \infty}^{r}} \leq C\left(\|u\|_{L^{\infty}}\|v\|_{B_{p, \infty}^{r}}+\|u\|_{B_{p, \infty}^{r}}\|v\|_{L^{\infty}}\right),
$$

where $C$ is constant depending on $r$ and $p$ only.

In the course of establishing existence results for the QG equation, very often we need to bound the $L^{\infty}$-norm of a function in terms of its norm in $B_{p, \infty}^{r}$. The following logarithmic estimate is very helpful. 
Proposition 2.5. Let $p \in[1, \infty]$, let $r_{c}=\frac{d}{p}$, and let $r>\frac{d}{p}$. Then there exists a constant $C$ depending on $p$ and $r$ only such that

$$
\|f\|_{L^{\infty}\left(\mathbb{R}^{d}\right)} \leq C\|f\|_{B_{p, \infty}^{r_{c}\left(\mathbb{R}^{d}\right)}} \log _{2}\left(e+\frac{\|f\|_{B_{p, \infty}^{r}\left(\mathbb{R}^{d}\right)}}{\|f\|_{B_{p, \infty}^{r_{c}}\left(\mathbb{R}^{d}\right)}}\right),
$$

which, in particular, implies

$$
\|f\|_{L^{\infty}\left(\mathbb{R}^{d}\right)} \leq C\|f\|_{B_{p, \infty}^{r}\left(\mathbb{R}^{d}\right)} .
$$

Proof. According to the definition of $\Delta_{i}$ 's in (2.1), $\Delta_{k} \Delta_{j}=0$ if $|k-j| \geq 2$. For $j \geq 0$,

$$
\begin{aligned}
\left\|\Delta_{j} f\right\|_{L^{\infty}} & \leq \sum_{|k-j| \leq 1}\left\|\Delta_{k} \Delta_{j} f\right\|_{L^{\infty}}=\sum_{|k-j| \leq 1}\left\|2^{k d} g\left(2^{k} \cdot\right) *\left(\Delta_{j} f\right)\right\|_{L^{\infty}} \\
& \leq \sum_{|k-j| \leq 1}\left\|2^{k d} g\left(2^{k} \cdot\right)\right\|_{L^{q}}\left\|\Delta_{j} f\right\|_{L^{p}}=\sum_{|k-j| \leq 1} 2^{k d \frac{1}{p}}\|g\|_{L^{q}}\left\|\Delta_{j} f\right\|_{L^{p}}
\end{aligned}
$$

where $q$ is the conjugate of $p$, or $1 / p+1 / q=1$. Thus,

$$
\left\|\Delta_{j} f\right\|_{L^{\infty}} \leq C 2^{j r_{c}}\left\|\Delta_{j} f\right\|_{L^{p}}
$$

for a pure constant $C$. A similar estimate for the case $j=-1$ leads to the same bound. Using this bound, we have

$$
\begin{aligned}
\|f\|_{L^{\infty}} & \leq \sum_{j \geq-1}\left\|\Delta_{j} f\right\|_{L^{\infty}}=\sum_{j=-1}^{N-1}\left\|\Delta_{j} f\right\|_{L^{\infty}}+\sum_{j \geq N}\left\|\Delta_{j} f\right\|_{L^{\infty}} \\
& \leq C(N+1)\|f\|_{B_{p}^{r_{c}}}+C\|f\|_{B_{p, \infty}^{r}} \sum_{j \geq N} 2^{-j\left(r-r_{c}\right)} \\
& =C(N+1)\|f\|_{B_{p, \infty}^{r_{c}}}+C \frac{2^{-N\left(r-r_{c}\right)}}{1-2^{-\left(r-r_{c}\right)}}\|f\|_{B_{p, \infty}^{r}} .
\end{aligned}
$$

The desired inequality (2.5) is then obtained by letting

$$
N=1+\left[\frac{1}{r-r_{c}} \log _{2} \frac{\|f\|_{B_{p, \infty}^{r}}}{\|f\|_{B_{p, \infty}^{r_{c}}}}\right] .
$$

Inequality (2.6) is true because of (2.5) and the fact that $x \rightarrow x \log _{2}(e+M / x)$ with a fixed constant $M$ is an increasing function for $x>0$. This completes the proof.

As seen in (1.1) of the introduction, the components of the velocity field $u$ are Riesz transforms of $\theta$, namely,

$$
u=\mathcal{R}^{\perp}(\theta) \equiv\left(-\mathcal{R}_{2}(\theta), \mathcal{R}_{1}(\theta)\right),
$$

where $\mathcal{R}_{k}=\partial_{x_{k}} \Lambda^{-1}$ for $k=1,2$ and $\Lambda \equiv(-\Delta)^{1 / 2}$. It is a classical result in the Calderon-Zygmund theory that for any $p \in(1, \infty)$ and $r \in \mathbb{R}$

$$
\|u\|_{B_{p, \infty}^{r}} \leq C\|\theta\|_{B_{p, \infty}^{r}},
$$

where $C$ is a constant depending only on $p$ and $r$. 
Finally, we recall the $L^{p}$-decay result of A. Córdoba and D. Córdoba. In a recent work [9], A. Córdoba and D. Córdoba skillfully proved a pointwise inequality involving the operator $\Lambda^{2 \alpha}$ with $\alpha \in[0,1]$ and then derived the $L^{p}$-decay result as a special consequence.

Proposition 2.6. Let $\alpha \in[0,1]$ and let $\theta \in \mathbb{S}$, the Schwartz class. Then,

$$
2 \theta \Lambda^{2 \alpha} \theta(x) \geq \Lambda^{2 \alpha} \theta^{2}(x)
$$

for any $x \in \mathbb{R}^{2}$.

The estimate in the following proposition is slightly different from the corresponding $L^{p}$-decay result derived in [9].

Proposition 2.7. Let $p=2^{k}$ for an integer $k \geq 1$. If $\theta$ solves (1.1) with an initial data $\theta_{0} \in L^{p}$, then the $L^{p}$-norm of $\theta$ decays algebraically in time. More precisely,

$$
\|\theta(\cdot, t)\|_{L^{p}} \leq \frac{\left\|\theta_{0}\right\|_{L^{p}}}{\left(1+\kappa C_{p} \gamma t\left\|\theta_{0}\right\|_{L^{2}}^{-\gamma p}\left\|\theta_{0}\right\|_{L^{p}}^{\gamma p}\right)^{\frac{1}{\gamma p}}},
$$

where $\gamma=\frac{\alpha}{p-2}$ and $C_{p}$ is a constant depending on $p$ and $\alpha$ only.

3. Global existence in $\boldsymbol{B}_{2, \infty}^{r}$. We shall assume in this section that $\theta_{0}$ is in the Besov space $B_{2, \infty}^{r}$. Consider the solution of the $2 \mathrm{D}$ dissipative QG equation

$$
\partial_{t} \theta+u \cdot \nabla \theta+\kappa \Lambda^{2 \alpha} \theta=0, \quad u=\mathcal{R}^{\perp}(\theta)
$$

with $\theta(x, 0)=\theta_{0}(x)$. Assuming $r>2-2 \alpha$, our major result states that (3.1) has a unique global solution if the norm of $\theta_{0}$ in $B_{2, \infty}^{r}$ is comparable to $\kappa$.

Theorem 3.1. Let $\kappa>0$ and let $0 \leq \alpha \leq \frac{1}{2}$. Assume the initial datum $\theta_{0}$ is in the Besov space $B_{2, \infty}^{r}$ with $r>2-2 \alpha$. There exists a constant $C_{0}$ depending on $\alpha$ and $r$ only such that if

$$
\left\|\theta_{0}\right\|_{B_{2, \infty}^{r}} \leq C_{0} \kappa
$$

then the $2 D$ dissipative $Q G$ equation (3.1) with $\theta(x, 0)=\theta_{0}(x)$ has a unique global solution $\theta$ satisfying

$$
\theta \in L^{\infty}\left([0, \infty) ; B_{2, \infty}^{r}\right) \cap L^{1}\left([0, \infty) ; B_{2, \infty}^{r+2 \alpha}\right) \cap \operatorname{Lip}\left([0, \infty) ; B_{2, \infty}^{r-1}\right) \cap C\left([0, \infty) ; B_{2, \infty}^{\delta}\right)
$$

for any $\delta \in[r-1, r)$, and

$$
\|\theta(\cdot, t)\|_{B_{2, \infty}^{r}} \leq C_{0} \kappa \quad \text { for any } t \geq 0
$$

Remark. Because of the embeddings

$$
B_{2,1}^{s} \hookrightarrow H_{2}^{s} \hookrightarrow B_{2, \infty}^{s},
$$

this theorem also implies that (3.1) has global solutions for small data in $B_{2,1}^{s}$ or $H^{s}$ with any $s>2-2 \alpha$.

Before proving Theorem 3.1, we first establish an a priori estimate.

Proposition 3.2. Assume that $\theta$ solves the $2 D$ dissipative $Q G$ equation (3.1) with $\kappa>0$ and $0 \leq \alpha \leq 1$. Let $r \in \mathbb{R}$ and let $s>2$. Then

$$
\frac{d}{d t}\|\theta\|_{B_{2, \infty}^{r}}+C_{1} \kappa\|\theta\|_{B_{2, \infty}^{r+2 \alpha}} \leq C_{2}\|\theta\|_{B_{2, \infty}^{s}}\|\theta\|_{B_{2, \infty}^{r}},
$$

where $C_{1}$ and $C_{2}$ are constants depending on $r$ only. 
If $r>2-2 \alpha$, we can choose $s=r+2 \alpha$. Then, (3.3) reduces to

$$
\frac{d}{d t}\|\theta\|_{B_{2, \infty}^{r}}+C_{1} \kappa\|\theta\|_{B_{2, \infty}^{r+2 \alpha}} \leq C_{2}\|\theta\|_{B_{2, \infty}^{r+2 \alpha}}\|\theta\|_{B_{2, \infty}^{r}} .
$$

This inequality bears two consequences, which we state as a corollary.

Corollary 3.3. Assume that $\theta$ solves the $2 D$ dissipative $Q G$ equation (3.1) with $\kappa>0$ and $0 \leq \alpha \leq 1$. Let $r>2-2 \alpha$ be a real number. There exists a constant $C_{0}$ depending on $\alpha$ and $r$ only such that if

$$
\left\|\theta_{0}\right\|_{B_{2, \infty}^{r}} \leq C_{0} \kappa
$$

then, for any $t \geq 0$,

$$
\|\theta(\cdot, t)\|_{B_{2, \infty}^{r}} \leq\left\|\theta_{0}\right\|_{B_{2, \infty}^{r}} \leq C_{0} \kappa .
$$

In addition, $\theta$ also satisfies the inequality

$$
\|\theta(\cdot, t)\|_{B_{2, \infty}^{r}}+C_{1} \kappa \int_{0}^{t}\|\theta(\cdot, \tau)\|_{B_{2, \infty}^{r+2 \alpha}} d \tau \leq\left\|\theta_{0}\right\|_{B_{2, \infty}^{r}} \exp \left(C_{2} \int_{0}^{t}\|\theta(\cdot, \tau)\|_{B_{2, \infty}^{r+2 \alpha}} d \tau\right) .
$$

Proof of Proposition 3.2. Let $j \geq-1$. Applying $\Delta_{j}$ to (3.1), we obtain

$$
\partial_{t} \Delta_{j} \theta+u \cdot \nabla \Delta_{j} \theta+\kappa \Lambda^{2 \alpha} \Delta_{j} \theta=\left[u \cdot \nabla, \Delta_{j}\right] \theta .
$$

Multiplying both sides by $2 \Delta_{j} \theta$ and integrating over $\mathbb{R}^{2}$ yields

$$
\frac{d}{d t} \int\left|\Delta_{j} \theta\right|^{2} d x+2 \kappa \int\left|\Lambda^{\alpha} \Delta_{j} \theta\right|^{2} d x=2 \int \Delta_{j} \theta\left[u \cdot \nabla, \Delta_{j}\right] \theta d x .
$$

Applying Lemma 2.1 to the dissipative term and Hölder's inequality to the right-hand side, we find that

$$
\frac{d}{d t}\left\|\Delta_{j} \theta\right\|_{L^{2}}+C \kappa 2^{2 \alpha j}\left\|\Delta_{j} \theta\right\|_{L^{2}} \leq\left\|\left[u \cdot \nabla, \Delta_{j}\right] \theta\right\|_{L^{2}} .
$$

In the above inequality, we have used the fact that Lemma 2.1 is valid for fractional derivatives when $p=2$. For any $r \in \mathbb{R}$, Proposition 2.2 applied to the term on the right-hand side yields

$$
\frac{d}{d t}\|\theta\|_{B_{2, \infty}^{r}}+C \kappa\|\theta\|_{B_{2, \infty}^{r+2 \alpha}} \leq C\left(\|\nabla u\|_{L^{\infty}}\|\theta\|_{B_{2, \infty}^{r}}+\|\nabla \theta\|_{L^{\infty}}\|u\|_{B_{2, \infty}^{r}}\right) .
$$

Furthermore, Proposition 2.5 applied to $\nabla u$ and $\nabla \theta$ asserts that for any $s>2$,

$$
\|\nabla u\|_{L^{\infty}} \leq C\|u\|_{B_{2, \infty}^{s}}, \quad\|\nabla \theta\|_{L^{\infty}} \leq C\|\theta\|_{B_{2, \infty}^{s}} .
$$

Inserting these estimates in (3.4) and noticing (2.7), we obtain

$$
\frac{d}{d t}\|\theta\|_{B_{2, \infty}^{r}}+C_{1} \kappa\|\theta\|_{B_{2, \infty}^{r+2 \alpha}} \leq C_{2}\|\theta\|_{B_{2, \infty}^{s}}\|\theta\|_{B_{2, \infty}^{r}} .
$$


Proof of Theorem 3.1. We start with a successive approximation sequence $\left\{\theta^{(n)}\right\}$ satisfying

$$
\left\{\begin{array}{l}
\theta^{(1)}=S_{2} \theta_{0}, \\
\partial_{t} \theta^{(n+1)}+u^{(n)} \cdot \nabla \theta^{(n+1)}+\kappa \Lambda^{2 \alpha} \theta^{(n+1)}=0, \\
u^{(n)}=\mathcal{R}^{\perp}\left(\theta^{(n)}\right), \\
\theta^{(n+1)}(x, 0)=\theta_{0}^{(n+1)}(x)=S_{n+2} \theta_{0} .
\end{array}\right.
$$

The rest of the proof is divided into two major parts. The first part establishes that $\left\{\theta^{(n)}\right\}$ is bounded uniformly in $L^{\infty}\left([0, \infty) ; B_{2, \infty}^{r}\right)$. The second part verifies that $\left\{\theta^{(n)}\right\}$ is a Cauchy sequence in $L^{\infty}\left([0, \infty) ; B_{2, \infty}^{r-1}\right)$.

Noticing that $r>2-2 \alpha$, we proceed as in the proof of Proposition 3.2 to obtain

$$
\begin{aligned}
\frac{d}{d t}\left\|\theta^{(n+1)}\right\|_{B_{2, \infty}^{r}} & +C C_{1} \kappa\left\|\theta^{(n+1)}\right\|_{B_{2, \infty}^{r+2 \alpha}} \\
& \leq C\left(\left\|\nabla u^{(n)}\right\|_{L^{\infty}}\left\|\theta^{(n+1)}\right\|_{B_{2, \infty}^{r}}+\left\|\nabla \theta^{(n+1)}\right\|_{L^{\infty}}\left\|u^{(n)}\right\|_{B_{2, \infty}^{r}}\right) \\
& \leq C\left(\left\|u^{(n)}\right\|_{B_{2, \infty}^{r+2 \alpha}}\left\|\theta^{(n+1)}\right\|_{B_{2, \infty}^{r}}+\left\|\theta^{(n+1)}\right\|_{B_{2, \infty}^{r+2 \alpha}}\left\|u^{(n)}\right\|_{B_{2, \infty}^{r}}\right) \\
& \leq C_{2}\left(\left\|\theta^{(n)}\right\|_{B_{2, \infty}^{r+2 \alpha}}\left\|\theta^{(n+1)}\right\|_{B_{2, \infty}^{r}}+\left\|\theta^{(n+1)}\right\|_{B_{2, \infty}^{r+2 \alpha}}\left\|\theta^{(n)}\right\|_{B_{2, \infty}^{r}}\right),
\end{aligned}
$$

where $C_{1}$ and $C_{2}$ are constants with dependence on $\alpha$ and $r$ only. Now, we choose $C_{0}<C_{1} /\left(4 C_{2}\right)$. Further restrictions will be imposed on $C_{0}$ in the second part. We show that if

$$
\left\|\theta_{0}\right\|_{B_{2, \infty}^{r}} \leq C_{0} \kappa
$$

then for any integer $n$ and any $t \geq 0$,

$$
\sup _{\tau \in[0, t]}\left\|\theta^{(n)}(\cdot, \tau)\right\|_{B_{2, \infty}^{r}}+C_{1} \kappa \int_{0}^{t}\left\|\theta^{(n)}(\cdot, \tau)\right\|_{B_{2, \infty}^{r+2 \alpha}} d \tau \leq 2 C_{0} \kappa .
$$

We proceed by induction. If (3.6) holds for $n=k$, namely,

$$
\left\|\theta^{(k)}(\cdot, t)\right\|_{B_{2, \infty}^{r}}+C_{1} \kappa \int_{0}^{t}\left\|\theta^{(k)}(\cdot, \tau)\right\|_{B_{2, \infty}^{r+2 \alpha}} d \tau \leq 2 C_{0} \kappa,
$$

then, according to (3.5),

$$
\begin{aligned}
\sup _{\tau \in[0, t]}\left\|\theta^{(k+1)}(\cdot, \tau)\right\|_{B_{2, \infty}^{r}}+C_{1} \kappa \int_{0}^{t}\left\|\theta^{(k+1)}(\cdot, \tau)\right\|_{B_{2, \infty}^{r+2 \alpha}} d \tau \\
\leq\left\|\theta_{0}^{(k+1)}\right\|_{B_{2, \infty}^{r}}+C_{2} \sup _{\tau \in[0, t]}\left\|\theta^{(k+1)}(\cdot, \tau)\right\|_{B_{2, \infty}^{r}} \int_{0}^{t}\left\|\theta^{(k)}(\cdot, \tau)\right\|_{B_{2, \infty}^{r+2 \alpha}} d \tau \\
\quad+C_{2} \sup _{\tau \in[0, t]}\left\|\theta^{(k)}(\cdot, \tau)\right\|_{B_{2, \infty}^{r}} \int_{0}^{t}\left\|\theta^{(k+1)}(\cdot, \tau)\right\|_{B_{2, \infty}^{r+2 \alpha}} d \tau \\
\leq\left\|\theta_{0}\right\|_{B_{2, \infty}^{r}}+\frac{2 C_{0} C_{2}}{C_{1}} \sup _{\tau \in[0, t]}\left\|\theta^{(k+1)}(\cdot, \tau)\right\|_{B_{2, \infty}^{r}} \\
\quad+2 C_{0} C_{2} \kappa \int_{0}^{t}\left\|\theta^{(k+1)}(\cdot, \tau)\right\|_{B_{2, \infty}^{r+2 \alpha}} d \tau .
\end{aligned}
$$


Since $2 C_{0} C_{2} \leq \frac{1}{2} C_{1}$, the inequality above becomes

$$
\sup _{\tau \in[0, t]}\left\|\theta^{(k+1)}(\cdot, \tau)\right\|_{B_{2, \infty}^{r}}+C_{1} \kappa \int_{0}^{t}\left\|\theta^{(k+1)}(\cdot, \tau)\right\|_{B_{2, \infty}^{r+2 \alpha}} d \tau \leq 2\left\|\theta_{0}\right\|_{B_{2, \infty}^{r}} \leq 2 C_{0} \kappa .
$$

Thus, (3.6) is verified. This completes the first part of the proof.

Next, we consider the difference

$$
\eta^{(n+1)}=\theta^{(n+1)}-\theta^{(n)} .
$$

The sequence $\left\{\eta^{(n)}\right\}$ satisfies

$$
\left\{\begin{array}{l}
\eta^{(1)}=S_{2} \theta_{0}-\theta_{0}, \\
\partial_{t} \eta^{(n+1)}+u^{(n)} \cdot \nabla \eta^{(n+1)}+\kappa \Lambda^{2 \alpha} \eta^{(n+1)}=w^{(n)} \cdot \nabla \theta^{(n)} \\
w^{(n)}=\mathcal{R}^{\perp}\left(\eta^{(n)}\right) \\
\eta^{(n+1)}(x, 0)=\eta_{0}^{(n+1)}(x)=\Delta_{n+1} \theta_{0} .
\end{array}\right.
$$

Starting with the equation for $\eta^{(n+1)}$ and proceeding as above, we are led to the following inequality:

$$
\begin{aligned}
& \frac{d}{d t}\left\|\eta^{(n+1)}\right\|_{B_{2, \infty}^{r-1}}+C_{1} \kappa\left\|\eta^{(n+1)}\right\|_{B_{2, \infty}^{r-1+2 \alpha}} \\
& \quad \leq 2^{(r-1) j}\left\|\left[u^{(n)} \cdot \nabla, \Delta_{j}\right] \eta^{(n+1)}\right\|_{L^{2}}+\left\|w^{(n)} \cdot \nabla \theta^{(n)}\right\|_{B_{2, \infty}^{r-1}} .
\end{aligned}
$$

Applying Propositions 2.3 and 2.5 to the first term on the right leads to

$$
\begin{aligned}
2^{(r-1) j}\left\|\left[u^{(n)} \cdot \nabla, \Delta_{j}\right] \eta^{(n+1)}\right\|_{L^{2}} & \leq C\left(\left\|\nabla u^{(n)}\right\|_{L^{\infty}}\left\|\eta^{(n+1)}\right\|_{B_{2, \infty}^{r-1}}+\left\|\eta^{(n+1)}\right\|_{L^{\infty}}\left\|u^{(n)}\right\|_{B_{2, \infty}^{r}}\right) \\
& \leq C\left(\left\|u^{(n)}\right\|_{B_{2, \infty}^{r+2 \alpha}}\left\|\eta^{(n+1)}\right\|_{B_{2, \infty}^{r-1}}+\left\|\eta^{(n+1)}\right\|_{B_{2, \infty}^{r-1+2 \alpha}}\left\|u^{(n)}\right\|_{B_{2, \infty}^{r}}\right) \\
& \leq C\left(\left\|\theta^{(n)}\right\|_{B_{2, \infty}^{r+2 \alpha}}\left\|\eta^{(n+1)}\right\|_{B_{2, \infty}^{r-1}}+\left\|\eta^{(n+1)}\right\|_{B_{2, \infty}^{r-1+2 \alpha}}\left\|\theta^{(n)}\right\|_{B_{2, \infty}^{r}}\right) .
\end{aligned}
$$

Since $\alpha \leq \frac{1}{2}, r-1>1-2 \alpha>0$ and the same estimate in Proposition 2.4 applies. Consequently,

$$
\begin{aligned}
& \left\|w^{(n)} \cdot \nabla \theta^{(n)}\right\|_{B_{2, \infty}^{r-1}} \leq C\left(\left\|w^{(n)}\right\|_{L^{\infty}}\left\|\nabla \theta^{(n)}\right\|_{B_{2, \infty}^{r-1}}+\left\|w^{(n)}\right\|_{B_{2, \infty}^{r-1}}\left\|\nabla \theta^{(n)}\right\|_{L^{\infty}}\right) \\
& \leq C\left(\left\|w^{(n)}\right\|_{B_{2, \infty}^{r-1+2 \alpha}}\left\|\theta^{(n)}\right\|_{B_{2, \infty}^{r}}+\left\|w^{(n)}\right\|_{B_{2, \infty}^{r-1}}\left\|\theta^{(n)}\right\|_{B_{2, \infty}^{r+2 \alpha}}\right)
\end{aligned}
$$

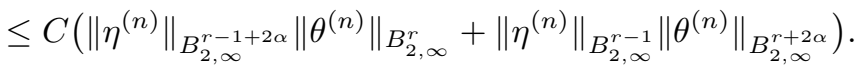

Inserting these estimates in (3.7) yields

$$
\begin{aligned}
\frac{d}{d t}\left\|\eta^{(n+1)}\right\|_{B_{2, \infty}^{r-1}}+C_{1} \kappa\left\|\eta^{(n+1)}\right\|_{B_{2, \infty}^{r-1+2 \alpha}} & \\
\leq & C_{3}\left(\left\|\theta^{(n)}\right\|_{B_{2, \infty}^{r+2 \alpha}}\left\|\eta^{(n+1)}\right\|_{B_{2, \infty}^{r-1}}+\left\|\eta^{(n+1)}\right\|_{B_{2, \infty}^{r-1+2 \alpha}}\left\|\theta^{(n)}\right\|_{B_{2, \infty}^{r}}\right) \\
& +C_{3}\left(\left\|\eta^{(n)}\right\|_{B_{2, \infty}^{r-1+2 \alpha}}\left\|\theta^{(n)}\right\|_{B_{2, \infty}^{r}}+\left\|\eta^{(n)}\right\|_{\left.B_{2, \infty}^{r-1}\left\|\theta^{(n)}\right\|_{B_{2, \infty}^{r+2 \alpha}}\right) .}\right.
\end{aligned}
$$


Integrating over $[0, t]$, we obtain

$$
\begin{aligned}
& \sup _{\tau \in[0, t]}\left\|\eta^{(n+1)}(\cdot, \tau)\right\|_{B_{2, \infty}^{r-1}}+C_{1} \kappa \int_{0}^{t}\left\|\eta^{(n+1)}(\cdot, \tau)\right\|_{B_{2, \infty}^{r-1+2 \alpha}} d \tau \\
& \quad \leq\left\|\theta_{0}^{(n+1)}\right\|_{B_{2, \infty}^{r-1}}+C_{3}\left(\sup _{\tau \in[0, t]}\left\|\eta^{(n+1)}\right\|_{B_{2, \infty}^{r-1}}+\sup _{\tau \in[0, t]}\left\|\eta^{(n)}\right\|_{B_{2, \infty}^{r-1}}\right) \int_{0}^{t}\left\|\theta^{(n)}\right\|_{B_{2, \infty}^{r+2 \alpha}} d \tau \\
& (3.8)+C_{3} \sup _{\tau \in[0, t]}\left\|\theta^{(n)}\right\|_{B_{2, \infty}^{r}} \int_{0}^{t}\left(\left\|\eta^{(n)}\right\|_{B_{2, \infty}^{r-1+2 \alpha}}+\left\|\eta^{(n+1)}\right\|_{B_{2, \infty}^{r-1+2 \alpha}}\right) d \tau .
\end{aligned}
$$

We now show by induction that for any $t \geq 0$,

$$
\sup _{\tau \in[0, t]}\left\|\eta^{(n)}(\cdot, \tau)\right\|_{B_{2, \infty}^{r-1}}+C_{1} \kappa \int_{0}^{t}\left\|\eta^{(n)}(\cdot, \tau)\right\|_{B_{2, \infty}^{r-1+2 \alpha}} d \tau \leq\left\|\theta_{0}\right\|_{B_{2, \infty}^{r}} 2^{-(n-3)} .
$$

First, we notice that

$$
\left\|\theta_{0}^{(n+1)}\right\|_{B_{2, \infty}^{r-1}}=\left\|\Delta_{n+1} \theta_{0}\right\|_{B_{2, \infty}^{r-1}} \leq\left\|\theta_{0}\right\|_{B_{2, \infty}^{r}} 2^{-n} .
$$

Now, we require that $C_{0}$ further satisfy

$$
2 C_{0} C_{3} / C_{1} \leq 1 / 4 \text {. }
$$

According to (3.6), we have the uniform bounds

$$
C_{3} \sup _{\tau \in[0, t]}\left\|\theta^{(n)}(\cdot, \tau)\right\|_{B_{2, \infty}^{r}} \leq 2 C_{0} C_{3} \kappa, \quad C_{3} \int_{0}^{t}\left\|\theta^{(n)}(\cdot, \tau)\right\|_{B_{2, \infty}^{r+2 \alpha}} d \tau \leq 2 C_{0} C_{3} / C_{1} .
$$

If (3.9) is satisfied by $n=k$, then it follows from (3.8) that

$$
\begin{aligned}
& \frac{3}{4}\left(\max _{\tau \in[0, t]}\left\|\eta^{(k+1)}(\cdot, \tau)\right\|_{B_{2, \infty}^{r-1}}+C_{1} \kappa \int_{0}^{t}\left\|\eta^{(k+1)}(\cdot, \tau)\right\|_{B_{2, \infty}^{r-1+2 \alpha}} d \tau\right) \\
& \quad \leq\left\|\theta_{0}\right\|_{B_{2, \infty}^{r}} 2^{-k}+\frac{1}{4}\left(\max _{\tau \in[0, t]}\left\|\eta^{(k)}(\cdot, \tau)\right\|_{B_{2, \infty}^{r-1}}+C_{1} \kappa \int_{0}^{t}\left\|\eta^{(k)}(\cdot, \tau)\right\|_{B_{2, \infty}^{r-1+2 \alpha}} d \tau\right) \\
& \quad \leq 3\left\|\theta_{0}\right\|_{B_{2, \infty}^{r}} 2^{-k} .
\end{aligned}
$$

Thus, (3.9) is true for $n=k+1$. In other words, $\left\{\eta^{(n)}\right\}=\left\{\theta^{(n)}-\theta^{(n-1)}\right\}$ is a Cauchy sequence in $L^{\infty}\left([0, \infty) ; B_{2, \infty}^{r-1}\right)$.

Therefore, there exists a $\theta \in L^{\infty}\left([0, \infty) ; B_{2, \infty}^{r}\right) \cap L^{1}\left([0, \infty) ; B_{2, \infty}^{r+2 \alpha}\right)$ such that

$$
\theta^{(n)} \rightarrow \theta \quad \text { in } \quad L^{\infty}\left([0, \infty) ; B_{2, \infty}^{r-1}\right) \cap L^{1}\left([0, \infty) ; B_{2, \infty}^{r-1+2 \alpha}\right) .
$$

Furthermore, for $0 \leq \alpha \leq \frac{1}{2}$,

$$
\begin{aligned}
\left\|\partial_{t} \theta^{(n)}(\cdot, t)\right\|_{B_{2, \infty}^{r-1}} & \leq\left\|u^{(n-1)} \cdot \nabla \theta^{(n)}(\cdot, t)\right\|_{B_{2, \infty}^{r-1}}+\kappa\left\|\Lambda^{2 \alpha} \theta^{(n)}(\cdot, t)\right\|_{B_{2, \infty}^{r-1}} \\
& \leq C_{3}\left\|\theta^{(n)}(\cdot, t)\right\|_{B_{2, \infty}^{r}}\left\|\theta^{(n-1)}(\cdot, t)\right\|_{B_{2, \infty}^{r-1}}+\kappa\left\|\theta^{(n)}(\cdot, t)\right\|_{B_{2, \infty}^{r}} \\
& \leq C_{3}\left(C_{0} \kappa\right)^{2}+C_{0} \kappa^{2}=\left(C_{3} C_{0}+1\right) C_{0} \kappa^{2} .
\end{aligned}
$$


Therefore, $\theta \in \operatorname{Lip}\left([0, \infty) ; B_{2, \infty}^{r-1}\right)$. Another consequence is $\theta \in C\left([0, \infty) ; B_{2, \infty}^{\delta}\right)$ for any $\delta \in[r-1, r)$. Finally, the a priori estimates in Proposition 3.2 and Corollary 3.3 allow us to conclude that

$$
\|\theta(\cdot, t)\|_{B_{2, \infty}^{r}} \leq C_{0} \kappa .
$$

This completes the proof.

4. Global existence in $B_{p, \infty}^{r}$ with $\boldsymbol{p}>2$. Attention is now turned to the $2 \mathrm{D}$ dissipative $\mathrm{QG}$ equation

$$
\partial_{t} \theta+u \cdot \nabla \theta+\kappa \Lambda^{2 \alpha} \theta=0
$$

with $\theta(x, 0)=\theta_{0}(x)$ in the Besov space $B_{p, \infty}^{r}$. We have the following theorem.

TheOrem 4.1. Let $\kappa>0$ and let $0 \leq \alpha \leq \frac{1}{2}$. Consider the solution of the dissipative $Q G$ equation (4.1) corresponding to $\theta_{0} \in B_{2, \infty}^{r} \cap B_{p, \infty}^{r}$ with $p=2^{N}(N>1)$. Assume that

$$
\left\{\begin{array}{l}
r>1+\frac{2}{p} \text { and }\left\|\theta_{0}\right\|_{B_{2, \infty}^{r}} \leq C_{0} \kappa \quad \text { if }(1-2 \alpha) p \leq 2 \\
r>2-2 \alpha,\left\|\theta_{0}\right\|_{B_{2, \infty}^{r}} \leq C_{0} \kappa, \text { and }\left\|\theta_{0}\right\|_{B_{p, \infty}^{r}} \leq C_{0} \kappa \quad \text { if }(1-2 \alpha) p>2
\end{array}\right.
$$

where $C_{0}$ is a suitably chosen constant with dependence on $\alpha, r$, and $p$ only. Then the $2 D Q G$ equation (4.1) has a unique global solution $\theta$ satisfying

$$
\theta \in L^{\infty}\left([0, \infty) ; B_{p, \infty}^{r}\right) \cap L^{1}\left([0, \infty) ; B_{p, \infty}^{r+2 \alpha}\right) \cap \operatorname{Lip}\left([0, \infty) ; B_{p, \infty}^{r-1}\right) \cap C\left([0, \infty) ; B_{p, \infty}^{\delta}\right)
$$

for any $\delta \in[r-1, r)$, and

$$
\|\theta(\cdot, t)\|_{B_{p, \infty}^{r}} \leq \max \left\{\left\|\theta_{0}\right\|_{B_{p, \infty}^{r}}, \tilde{C}_{0} \kappa\right\}
$$

for any $t \geq 0$ and some constant $\tilde{C}_{0}$ depending on $\alpha, r$, and $p$ only.

The rest of this section revolves around the proof of Theorem 4.1 and is divided into two subsections. The first subsection presents an a priori estimate and the second subsection proves Theorem 4.1.

4.1. An a priori bound. We state and prove a global a priori bound.

Proposition 4.2. Assume that $\theta$ solves the $2 D$ dissipative $Q G$ equation (4.1) with $\kappa>0$ and $0 \leq \alpha \leq 1$. Let $r \in \mathbb{R}$, let $p=2^{N}$ for an integer $N>1$, and let $s>1+\frac{2}{p}$. Then

$$
\frac{d}{d t}\|\theta\|_{B_{p, \infty}^{r}}+C_{4} p^{-1} \kappa\|\theta\|_{B_{p, \infty}^{r}}^{1+\beta p}\|\theta\|_{B_{2, \infty}^{r}}^{-\beta p} \leq C_{5}\|\theta\|_{B_{p, \infty}^{r}}\|\theta\|_{B_{p, \infty}^{s}}
$$

where $\beta=\frac{2 \alpha}{p-2}$, and $C_{4}$ and $C_{5}$ are constants with possible dependence on $\alpha$ and $p$ only.

Remark. The case $p=2$ is excluded here since this case has been dealt with in the previous section. The assumption $p=2^{N}$ is made in order to use Proposition 2.7.

Proof of Proposition 4.2. Applying $\Delta_{j}$ to (4.1), multiplying by $p\left|\Delta_{j} \theta\right|^{p-2} \Delta_{j} \theta$, and integrating over $\mathbb{R}^{2}$, we obtain

$$
\frac{d}{d t}\left\|\Delta_{j} \theta\right\|_{L^{p}}^{p}+I=I I
$$


where $I$ and $I I$ represent the terms

$$
\begin{gathered}
I=\kappa p \int\left|\Delta_{j} \theta\right|^{p-2}\left(\Delta_{j} \theta\right) \Lambda^{2 \alpha} \Delta_{j} \theta d x, \\
I I=p \int\left|\Delta_{j} \theta\right|^{p-2}\left(\Delta_{j} \theta\right)\left[u \cdot \nabla, \Delta_{j}\right] \theta d x .
\end{gathered}
$$

To estimate $I I$, we first apply Hölder's inequality and then Proposition 2.2 to obtain

$$
\begin{aligned}
|I I| & \leq p\left\|\Delta_{j} \theta\right\|_{L^{p}}^{p-1}\left\|\left[u \cdot \nabla, \Delta_{j}\right] \theta\right\|_{L^{p}} \\
& \leq p\left\|\Delta_{j} \theta\right\|_{L^{p}}^{p-1}\left[2^{-j r}\left(\|\nabla u\|_{L^{\infty}}\|\theta\|_{B_{p, \infty}^{r}}+\|\nabla \theta\|_{L^{\infty}}\|u\|_{B_{p, \infty}^{r}}\right)\right] .
\end{aligned}
$$

For $s>1+\frac{2}{p}$, Proposition 2.5 asserts that

$$
\|\nabla \theta\|_{L^{\infty}} \leq C\|\theta\|_{B_{p, \infty}^{s}}, \quad\|\nabla u\|_{L^{\infty}} \leq C\|u\|_{B_{p}^{s}, \infty} \leq C\|\theta\|_{B_{p, \infty}^{s}} .
$$

Therefore, for some constant $C$,

$$
|I I| \leq C p 2^{-j r}\left\|\Delta_{j} \theta\right\|_{L^{p}}^{p-1}\|\theta\|_{B_{p, \infty}^{r}}\|\theta\|_{B_{p, \infty}^{s}} .
$$

To obtain a lower bound for $I$, we use Proposition 2.6 and a basic embedding inequality,

$$
I \geq C \kappa \int\left|\Lambda^{\alpha}\left(\left|\Delta_{j} \theta\right|^{\frac{p}{2}}\right)\right|^{2} d x \geq C \kappa\left(\int\left|\Delta_{j} \theta\right|^{\frac{p}{1-\alpha}} d x\right)^{1-\alpha}=C \kappa\left\|\Delta_{j} \theta\right\|_{L^{\frac{p}{1-\alpha}}}^{p},
$$

where the assumption $p=2^{N}$ is used in the first inequality. Applying the interpolation inequality

$$
\|f\|_{L^{p}} \leq C\|f\|_{L^{2}}^{\frac{2 \alpha}{p+2 \alpha-2}}\|f\|_{L^{\frac{p}{1-\alpha}}}^{\frac{p-2}{p+2 \alpha-2}}
$$

with $f=\Delta_{j} \theta$, we finally obtain the lower bound

$$
I \geq C \kappa\left\|\Delta_{j} \theta\right\|_{L^{p}}^{(1+\beta) p}\left\|\Delta_{j} \theta\right\|_{L^{2}}^{-\beta p},
$$

where we have set $\beta=\frac{2 \alpha}{p-2}$. Combining (4.4), (4.5), and (4.6) yields

$$
\frac{d}{d t}\|\theta\|_{B_{p, \infty}^{r}}+C p^{-1} \kappa 2^{j r}\left\|\Delta_{j} \theta\right\|_{L^{p}}^{1+\beta p}\left\|\Delta_{j} \theta\right\|_{L^{2}}^{-\beta p} \leq C\|\theta\|_{B_{p, \infty}^{r}}\|\theta\|_{B_{p, \infty}^{s}}
$$

or, equivalently,

$$
\frac{d}{d t}\|\theta\|_{B_{p, \infty}^{r}}+C p^{-1} \kappa\|\theta\|_{B_{p, \infty}^{r}}^{1+\beta p}\|\theta\|_{B_{2, \infty}^{r}}^{-\beta p} \leq C\|\theta\|_{B_{p, \infty}^{r}}\|\theta\|_{B_{p, \infty}^{s}} .
$$

We now explore several consequences of Proposition 4.2. If $(1-2 \alpha) p \leq 2$, then $2 \alpha+2 / p \geq 1$ or $\beta p \geq 1$. In addition, $r>1+2 / p$ implies $r>2-2 \alpha$. It thus follows from Corollary 3.3 that $\left\|\theta_{0}\right\|_{B_{2, \infty}^{r}} \leq C_{0} \kappa$ implies

$$
\|\theta(\cdot, t)\|_{B_{2, \infty}^{r}} \leq C_{0} \kappa
$$


for all $t>0$. Consequently, (4.3) can be reduced to

$$
\frac{d}{d t}\|\theta\|_{B_{p, \infty}^{r}} \leq C_{5}\|\theta\|_{B_{p, \infty}^{r}}^{2}\left(1-C_{5}^{-1} C_{4}\left(p C_{0}^{\beta p}\right)^{-1} \kappa^{1-\beta p}\|\theta\|_{B_{p, \infty}^{r}-1}^{\beta p-1}\right) .
$$

For $\beta p>1,(4.7)$ indicates that

$$
\left\{\begin{array}{l}
\|\theta(\cdot, t)\|_{B_{p, \infty}^{r}} \text { decreases as a function of } t \text { for a big initial norm }\left\|\theta_{0}\right\|_{B_{p, \infty}^{r}}, \\
\|\theta(\cdot, t)\|_{B_{p, \infty}^{r}} \text { increases up to }\left(C_{4} /\left(p C_{5} C_{0}^{\beta p}\right)\right)^{\frac{1}{\beta p-1}} \kappa \text { for small }\left\|\theta_{0}\right\|_{B_{p, \infty}^{r}} .
\end{array}\right.
$$

In other words,

$$
\|\theta(\cdot, t)\|_{B_{p, \infty}^{r}} \leq \max \left\{\left\|\theta_{0}\right\|_{B_{p, \infty}^{r}},\left(C_{4} /\left(p C_{5} C_{0}^{\beta p}\right)\right)^{\frac{1}{\beta p-1}} \kappa\right\} .
$$

For $\beta p=1$ and $C_{0} \leq C_{4} /\left(p C_{5}\right),(4.7)$ indicates that $\|\theta(\cdot, t)\|_{B_{p, \infty}^{r}}$ is a decreasing function of $t$ and thus

$$
\|\theta(\cdot, t)\|_{B_{p, \infty}^{r}} \leq\left\|\theta_{0}\right\|_{B_{p, \infty}^{r}}
$$

for any $t \geq 0$.

If $(1-2 \alpha) p>2$, then $1+\beta p<2$ and $r>2-2 \alpha$ implies that $r>1+\frac{2}{p}$. In this case, (4.3) becomes

$$
\frac{d}{d t}\|\theta\|_{B_{p, \infty}^{r}} \leq\|\theta\|_{B_{p, \infty}^{r}}^{1+\beta p}\left(C_{5}\|\theta\|_{B_{p, \infty}^{r}}^{1-\beta p}-C_{4}\left(p C_{0}^{\beta p}\right)^{-1} \kappa^{1-\beta p}\right) .
$$

If $\theta_{0}$ satisfies

$$
\left\|\theta_{0}\right\|_{B_{p, \infty}^{r}} \leq\left(C_{4} /\left(p C_{5} C_{0}^{\beta p}\right)\right)^{\frac{1}{1-\beta p}} \kappa
$$

then $\|\theta(\cdot, t)\|_{B_{p, \infty}^{r}}$ decreases as a function of $t$ and thus

$$
\|\theta(\cdot, t)\|_{B_{p, \infty}^{r}} \leq\left\|\theta_{0}\right\|_{B_{p, \infty}^{r}}
$$

for any $t \geq 0$.

In summary, we have established the following corollary.

Corollary 4.3. Let $\kappa>0$ and let $0 \leq \alpha \leq 1$. Assume that $\theta$ solves the $2 D$ dissipative $Q G$ equation (4.1) corresponding to $\theta_{0}$ in $B_{2, \infty}^{r} \cap B_{p, \infty}^{r}$ with $p=2^{N}(N>1)$. If $r$ and $\theta_{0}$ satisfy (4.2), then we have the global bounds

$$
\|\theta(\cdot, t)\|_{B_{2, \infty}^{r}} \leq \tilde{C}_{0} \kappa \quad \text { and } \quad\|\theta(\cdot, t)\|_{B_{p, \infty}^{r}} \leq \max \left\{\left\|\theta_{0}\right\|_{B_{p, \infty}^{r}}, \tilde{C}_{0} \kappa\right\}
$$

for some constant $\tilde{C}_{0}$ depending on $\alpha, r$, and $p$ only.

It is worth mentioning that the argument leading to the above corollary can be replaced by utilizing explicit formulas given in the following lemma.

Lemma 4.4. Let $\sigma>0$. Assume that $y=y(t)$ satisfies

$$
\frac{d}{d t} y+g(t) y^{1+\sigma} \leq h(t) y
$$


for real-valued functions $g$ and $h$. Then $y=y(t)$ is bounded pointwise according to

$$
y(t) \leq \frac{y(0) \exp \left(\int_{0}^{t} h(\tau) d \tau\right)}{\left(1+\sigma y^{\sigma}(0) \int_{0}^{t} g(\tau) \exp \left(\sigma \int_{0}^{\tau} h(s) d s\right) d \tau\right)^{\frac{1}{\sigma}}} .
$$

Proof. It follows easily from (4.9) that $z=y \exp \left(-\int_{0}^{t} h(\tau) d \tau\right)$ satisfies

$$
\frac{d}{d t} z \leq-g(t) \exp \left(\sigma \int_{0}^{t} h(\tau) d \tau\right) z^{1+\sigma}
$$

Dividing both sides by $z^{1+\sigma}$ and integrating over $[0, t]$, we obtain

$$
z^{-\sigma}(t) \geq z^{-\sigma}(0)+\sigma \int_{0}^{t} g(\tau) \exp \left(\sigma \int_{0}^{\tau} h(s) d s\right) d \tau,
$$

which can be converted into the following inequality for $y$ :

$$
y^{\sigma} \leq \frac{y^{\sigma}(0) \exp \left(\sigma \int_{0}^{t} h(\tau) d \tau\right)}{1+\sigma y^{\sigma}(0) \int_{0}^{t} g(\tau) \exp \left(\sigma \int_{0}^{\tau} h(s) d s\right) d \tau} .
$$

Raising both sides to $\frac{1}{\sigma}$ yields (4.10).

When an extra term $f(t)$ is added to (4.9), the method of variation of constants still allows us to obtain a formal bound involving a function $C(t)$, which satisfies an additional ordinary differential equation.

Lemma 4.5. Let $\sigma>0$. Assume that $y=y(t)$ satisfies

$$
\frac{d}{d t} y+g(t) y^{1+\sigma} \leq h(t) y+f(t)
$$

for real-valued functions $g, h$, and $f$. Then $y$ obeys the bound

$$
y(t) \leq \frac{\exp \left(\int_{0}^{t} h(\tau) d \tau\right)}{\left(-\sigma C(t)+\sigma \int_{0}^{t} g(\tau) \exp \left(\sigma \int_{0}^{\tau} h(s) d s\right) d \tau\right)^{\frac{1}{\sigma}}}
$$

where $C(t)$ satisfies the following ordinary differential equation:

$$
\begin{aligned}
\frac{d}{d t} C(t)= & f(t) \exp \left(-\int_{0}^{t} h(\tau) d \tau\right) \\
& \times\left(-\sigma C(t)+\sigma \int_{0}^{t} g(\tau) \exp \left(\sigma \int_{0}^{\tau} h(s) d s\right) d \tau\right)^{1+\frac{1}{\sigma}}
\end{aligned}
$$

with the initial datum $C(0)=-1 /\left(\sigma y^{\sigma}(0)\right)$.

Remark. When $f=0, C(t)=C(0)=-1 /\left(\sigma y^{\sigma}(0)\right)$ and (4.12) becomes (4.10). 
4.2. Proof of Theorem 4.1. Assume that $\left\{\theta^{(n)}\right\}$ is a successive approximation sequence satisfying the equations

$$
\left\{\begin{array}{l}
\theta^{(1)}=S_{2} \theta_{0}, \\
\partial_{t} \theta^{(n+1)}+u^{(n)} \cdot \nabla \theta^{(n+1)}+\kappa \Lambda^{2 \alpha} \theta^{(n+1)}=0, \\
u^{(n)}=\mathcal{R}^{\perp}\left(\theta^{(n)}\right), \\
\theta^{(n+1)}(x, 0)=\theta_{0}^{(n+1)}(x)=S_{n+2} \theta_{0} .
\end{array}\right.
$$

Following the same procedure as in the proof of Proposition 4.2 leads to

$\frac{d}{d t}\left\|\theta^{(n+1)}\right\|_{B_{p, \infty}^{r}}+C_{4} p^{-1} \kappa\left\|\theta^{(n+1)}\right\|_{B_{p, \infty}^{r}}^{1+\beta p}\left\|\theta^{(n+1)}\right\|_{B_{2, \infty}^{r}}^{-\beta p} \leq C_{5}\left\|\theta^{(n)}\right\|_{B_{p, \infty}^{r}}\left\|\theta^{(n+1)}\right\|_{B_{p, \infty}^{r}}$

If the conditions in (4.2) are met, we know from the proof of Theorem 3.1 that

$$
\left\|\theta^{(n)}(\cdot, t)\right\|_{B_{2, \infty}^{r}} \leq C_{0} \kappa
$$

for any integer $n$ and any $t \geq 0$. Inequality (4.14) can then be rewritten as

$$
\frac{d}{d t}\left\|\theta^{(n+1)}\right\|_{B_{p, \infty}^{r}} \leq\left\|\theta^{(n+1)}\right\|_{B_{p, \infty}^{r}}\left(C_{5}\left\|\theta^{(n)}\right\|_{B_{p, \infty}^{r}}-C_{4}\left(p C_{0}^{\beta p}\right)^{-1} \kappa^{1-\beta p}\left\|\theta^{(n+1)}\right\|_{B_{p, \infty}^{r}}^{\beta p}\right) .
$$

When (4.2) is satisfied, we can argue similarly as in the previous subsection and conclude that

$$
\left\|\theta^{(n)}(\cdot, t)\right\|_{B_{p, \infty}^{r}} \leq\left\{\begin{array}{l}
\left\|\theta_{0}\right\|_{B_{p, \infty}^{r}} \text { if } \beta p \leq 1 \\
\max \left\{\left\|\theta_{0}\right\|_{B_{p, \infty}^{r}},\left(C_{4} /\left(p C_{5} C_{0}^{\beta p}\right)\right)^{\frac{1}{\beta p-1}} \kappa\right\} \quad \text { if } \beta p>1 .
\end{array}\right.
$$

An alternative argument using the explicit formula in Lemma 4.9 also leads to the same bound.

We now show that $\left\{\eta^{(n)}\right\}=\left\{\theta^{(n)}-\theta^{(n-1)}\right\}$ is a Cauchy sequence in $C\left([0, \infty) ; B_{p, \infty}^{r-1}\right)$. The sequence $\left\{\eta^{(n)}\right\}$ satisfies

$$
\left\{\begin{array}{l}
\eta^{(1)}=S_{2} \theta_{0}-\theta_{0} \\
\partial_{t} \eta^{(n+1)}+u^{(n)} \cdot \nabla \eta^{(n+1)}+\kappa \Lambda^{2 \alpha} \eta^{(n+1)}=w^{(n)} \cdot \nabla \theta^{(n)} \\
w^{(n)}=\mathcal{R}^{\perp}\left(\eta^{(n)}\right) \\
\eta^{(n+1)}(x, 0)=\eta_{0}^{(n+1)}(x)=\Delta_{n+1} \theta_{0}
\end{array}\right.
$$

Following the procedures as in the proof of Theorem 3.1 as well as in the first part of this proof, we obtain

$$
\frac{d}{d t}\left\|\eta^{(n+1)}\right\|_{B_{p, \infty}^{r-1}}+C_{4} p^{-1} \kappa\left\|\eta^{(n+1)}\right\|_{B_{p, \infty}^{r-1}}^{1+\beta p}\left\|\eta^{(n+1)}\right\|_{B_{2, \infty}^{r-1}}^{-\beta p} \leq K_{1}+K_{2},
$$

where $K_{1}$ and $K_{2}$ represent

$$
K_{1}=2^{(r-1) j}\left\|\left[u^{(n)} \cdot \nabla, \Delta_{j}\right] \eta^{(n+1)}\right\|_{L^{p}}, \quad K_{2}=\left\|w^{(n)} \cdot \nabla \theta^{(n)}\right\|_{B_{p, \infty}^{r-1}}
$$


To estimate $K_{1}$ and $K_{2}$, we assume that (4.2) is satisfied. By Proposition 2.3,

$$
\begin{aligned}
K_{1} & \leq C\left(\left\|\nabla u^{(n)}\right\|_{L^{\infty}}\left\|\eta^{(n+1)}\right\|_{B_{p, \infty}^{r-1}}+\left\|\eta^{(n+1)}\right\|_{L^{\infty}}\left\|u^{(n)}\right\|_{B_{p, \infty}^{r}}\right) \\
& \leq 2 C\left\|u^{(n)}\right\|_{B_{p, \infty}^{r}}\left\|\eta^{(n+1)}\right\|_{B_{2, \infty}^{r-1}} \\
& \leq 2 C\left\|\theta^{(n)}\right\|_{B_{p, \infty}^{r}}\left\|\eta^{(n+1)}\right\|_{B_{p, \infty}^{r-1}} .
\end{aligned}
$$

By Proposition 2.4,

$$
\begin{aligned}
K_{2} & \leq C\left(\left\|w^{(n)}\right\|_{L^{\infty}}\left\|\nabla \theta^{(n)}\right\|_{B_{p, \infty}^{r-1}}+\left\|w^{(n)}\right\|_{B_{p, \infty}^{r-1}}\left\|\nabla \theta^{(n)}\right\|_{L^{\infty}}\right) \\
& \leq 2 C\left\|\theta^{(n)}\right\|_{B_{p, \infty}^{r}}\left\|w^{(n)}\right\|_{B_{p, \infty}^{r-1}} \\
& \leq 2 C\left\|\theta^{(n)}\right\|_{B_{p, \infty}^{r}}\left\|\eta^{(n)}\right\|_{B_{p, \infty}^{r-1}}
\end{aligned}
$$

Inserting (4.17) and (4.18) in (4.16), we obtain

$$
\begin{aligned}
& \frac{d}{d t}\left\|\eta^{(n+1)}\right\|_{B_{p, \infty}^{r-1}}+C_{4} p^{-1} \kappa\left\|\eta^{(n+1)}\right\|_{B_{p, \infty}^{r-1}}^{1+\beta p}\left\|\eta^{(n+1)}\right\|_{B_{2, \infty}^{r-1}}^{-\beta p} \\
& \quad \leq C_{7}\left\|\theta^{(n)}\right\|_{B_{p, \infty}^{r}}\left(\left\|\eta^{(n+1)}\right\|_{B_{p, \infty}^{r-1}}+\left\|\eta^{(n)}\right\|_{B_{p, \infty}^{r-1}}\right) .
\end{aligned}
$$

According to the proof of Theorem 3.1 and the first part of this proof,

$$
\left\|\eta^{(n+1)}\right\|_{B_{2, \infty}^{r-1}} \leq\left\|\theta_{0}\right\|_{B_{2, \infty}^{r-1}} 2^{-(n-2)}, \quad\left\|\theta^{(n)}\right\|_{B_{p, \infty}^{r}} \leq \max \left\{\left\|\theta_{0}\right\|_{B_{p, \infty}^{r}}, \tilde{C} \kappa\right\},
$$

where $\tilde{C}$ is a constant. We are now ready to show that

$$
\left\|\eta^{(n+1)}(\cdot, t)\right\|_{B_{p, \infty}^{r-1}} \leq \bar{C} 2^{-(n-2-1 /(\beta p))},
$$

where $\bar{C}$ is given explicitly by

$$
\bar{C}=\max \left\{\frac{1}{2},\left(2 C_{7} \max \left\{\left\|\theta_{0}\right\|_{B_{p, \infty}^{r}}, \tilde{C} \kappa\right\} /\left(C_{4} p^{-1} \kappa\right)\right)^{1 / \sigma}\right\}\left\|\theta_{0}\right\|_{B_{p, \infty}^{r}} .
$$

To simplify the notation, we set

$$
\begin{gathered}
\sigma=\beta p, \quad y(t)=\left\|\eta^{(n+1)}(\cdot, t)\right\|_{B_{p, \infty}^{r-1}}, \quad g=C_{4} p^{-1} \kappa 2^{(n-1)}\left\|\theta_{0}\right\|_{B_{2, \infty}^{r-1}}^{-\sigma}, \\
h=C_{7} \max \left\{\left\|\theta_{0}\right\|_{B_{p, \infty}^{r}}, \tilde{C} \kappa\right\}, \quad f=C_{7} \max \left\{\left\|\theta_{0}\right\|_{B_{p, \infty}^{r}}, \tilde{C} \kappa\right\} \bar{C} 2^{-(n-3-1 /(\beta p))} .
\end{gathered}
$$

Inequality (4.19) then becomes

$$
\frac{d}{d t} y \leq-g y^{\sigma+1}+h y+f .
$$

We further write $z(t)$ for the right-hand side of the inequality above. If $y(0)$ is sufficiently large such that $z(0) \leq 0$, then

$$
N \equiv \sup _{t \geq 0} y(t) \leq y(0)
$$


If, on the other hand, $y(0)$ is small and $z(0) \geq 0$, then $y(t)$ initially grows as $t$ increases. But its growth stops as soon as $z(t)$ becomes zero. Therefore, $N$ obeys

$$
-g N^{1+\sigma}+h N+f=0 \quad \text { or } \quad N^{1+\sigma}-\frac{h}{g} N=\frac{f}{g} .
$$

The discussion is then divided into two cases: i) $N^{\sigma} \leq 2 h / g$ and ii) $N^{\sigma}>2 h / g$. In the first case,

$$
N \leq\left(\frac{2 h}{g}\right)^{\frac{1}{\sigma}} .
$$

In the second case, (4.20) implies that

$$
N^{1+\sigma}<\frac{2 f}{g} \quad \text { or } \quad N \leq\left(\frac{2 f}{g}\right)^{\frac{1}{1+\sigma}} .
$$

In summary, we have obtained

$$
\sup _{t \geq 0} y(t) \leq \max \left\{y(0),\left(\frac{2 h}{g}\right)^{\frac{1}{\sigma}},\left(\frac{2 f}{g}\right)^{\frac{1}{1+\sigma}}\right\} .
$$

Returning to the original variable, we find

$$
\begin{gathered}
y(0)=\left\|\Delta_{n+1} \theta_{0}\right\|_{B_{p, \infty}^{r-1}} \leq\left\|\theta_{0}\right\|_{B_{p, \infty}^{r}} 2^{-n}, \quad\left(\frac{2 h}{g}\right)^{\frac{1}{\sigma}} \leq \bar{C} 2^{-n+2}, \\
\left(\frac{2 f}{g}\right)^{\frac{1}{1+\sigma}} \leq \bar{C}\left(2^{-n+3+1 / \sigma} 2^{-\sigma(n-2)}\right)^{\frac{1}{1+\sigma}} \leq \bar{C} 2^{-(n-2-1 / \sigma)} .
\end{gathered}
$$

As a consequence, (4.21) yields the desired bound

$$
\sup _{t \geq 0}\left\|\eta^{(n+1)}(\cdot, t)\right\|_{B_{p, \infty}^{r-1}} \leq \bar{C} 2^{-(n-2-1 /(\beta p))} .
$$

After a similar argument as in the proof of Theorem 3.1, the proof of Theorem 4.1 is then completed.

\section{REFERENCES}

[1] L. BerselLi, Vanishing viscosity limit and long-time behavior for $2 D$ quasi-geostrophic equations, Indiana Univ. Math. J., 51 (2002), pp. 905-930.

[2] D. ChaE, The quasi-geostrophic equation in the Triebel-Lizorkin spaces, Nonlinearity, 16 (2003), pp. 479-495.

[3] D. Chat And J. LeE, Global well-posedness in the super-critical dissipative quasi-geostrophic equations, Comm. Math. Phys., 233 (2003), pp. 297-311.

[4] J.-Y. Chemin, Perfect Incompressible Fluids, Clarendon Press, Oxford, UK, 1998.

[5] P. Constantin, A. Majda, And E. Tabak, Formation of strong fronts in the 2-D quasigeostrophic thermal active scalar, Nonlinearity, 7 (1994), pp. 1495-1533.

[6] P. Constantin, D. Córdoba, and J. Wu, On the critical dissipative quasi-geostrophic equation, Indiana Univ. Math. J., 50 (2001), pp. 97-107.

[7] P. Constantin And J. Wu, Behavior of solutions of $2 D$ quasi-geostrophic equations, SIAM J. Math. Anal., 30 (1999), pp. 937-948.

[8] D. CóRdobA, Nonexistence of simple hyperbolic blow-up for the quasi-geostrophic equation, Ann. of Math. (2), 148 (1998), pp. 1135-1152. 
[9] A. Córdoba And D. Córdoba, A maximum principle applied to quasi-geostrophic equations, Comm. Math. Phys., 249 (2004), pp. 511-528.

[10] D. Córdoba And C. Fefferman, Growth of solutions for $Q G$ and $2 D$ Euler equations, J. Amer. Math. Soc., 15 (2002), pp. 665-670.

[11] I. Held, R. Pierrehumbert, S. Garner, and K. Swanson, Surface quasi-geostrophic dynamics, J. Fluid Mech., 282 (1995), pp. 1-20.

[12] J. Pedlosky, Geophysical Fluid Dynamics, Springer-Verlag, New York, 1987.

[13] S. Resnick, Dynamical Problem in Nonlinear Advective Partial Differential Equations, Ph.D. thesis, University of Chicago, Chicago, 1995.

[14] M. E. SchonbeK AND T. P. SCHONBeK, Asymptotic behavior to dissipative quasi-geostrophic flows, SIAM J. Math. Anal., 35 (2003), pp. 357-375.

[15] J. Wu, Inviscid limits and regularity estimates for the solutions of the $2 D$ dissipative quasigeostrophic equations, Indiana Univ. Math. J., 46 (1997), pp. 1113-1124.

[16] J. Wu, Dissipative quasi-geostrophic equations with $L^{p}$ data, Electron. J. Differential Equations, 2001 (2001), pp. 1-13.

[17] J. Wu, Solutions of the 2D Quasi-geostrophic Equation in Hölder Spaces, preprint, Oklahoma State University, Stillwater, OK, 2003. 